THREE CASES OF PURPURA HEMORRHAGICA IN CHRONIC TUBERCULOSIS

WITH A BRIEF REVIEW *

S. BAUCH, M.D.

NEW YORK

The subject of purpura in this paper is treated from the standpoint of the relationship it bears to tuberculosis.

Several writers within the last two centuries have made observations on this phenomenon, endeavoring to find its cause and effect, and to discover the relation purpura has to other diseases with which it is associated.

Mackenzie ${ }^{1}$ says :

The great variety of supposed causes or associated conditions is sufficiently striking. Still more so is the fact that in one-third of 200 cases no explanation was offered for the purpura, though in several of the cases a necropsy was made. It will thus be seen how extremely complex is the pathology of purpura.

All we can do in the present state of our knowledge is to accumulate further information and to exhaust every means-histological, bacteriological and chemical-in the investigation of cases.

Osler" $^{2}$ also maintains "that purpura is obscure, and is an interesting manifestation of which we know so much and at the same time so little."

We may safely say, however, that the purpura observed in the three cases herein reported shows a striking relation between it and chronic tuberculosis. We may also claim that purpura is not of obscure origin, but that it has a definite mechanicotoxic cause.

The following are observations made by various authors who have given attention to this subject.

Osler claims that "purpura hemorrhagica rarely occurs with tuberculosis, but a fatal case has been reported."

Dieulafoy ${ }^{3}$ divides purpura into different groups according to the diseases with which it is associated, as follows:

1. The nervous, as in tabes, myelitis and cancer of the spine.

2. Medicinal, as in copaiba, belladonna, iodin and snake venom poisoning.

3. The rheumatoid, as in peliosis rheumatica of Schoenlein.

4. Infectious: variola, erysipelas, typhoid, septicemia, infective endocarditis and pyemia.

5. Necrotic purpura, as in gangrene.

6. Acute pulmonary tuberculosis.

* Submitted for publication Dec. 4, 1915.

* From the Tuberculosis Department of the Montefiore Home and Hospital.

1. Allbutt's System of Medicine, vi.

2. Osler's Modern Medicine, iv.

3. Dieulafoy: Principles of Medicine. 
Struempel1 ${ }^{4}$ endeavors in his classification to show that purpura has its origin in an infection of some sort. Rosenow of Chicago holds the same view. Struempell claims that if rabbits are inoculated with the blood of a patient having purpura the animal will show similar cutaneous symptoms. This tends to show that whether it is in typhoid, septic endocarditis or rheumatic fever there is some bacillus or coccus that is the direct etiological factor.

Adami5 attributes the causes of all purpura to a poison which acts on the endothelium of the smaller cutaneous vessels, producing fatty degeneration. It is the weakening and necrosis of these cells that would seem to precede the hemorrhage by rhexis or diapedesis which set up the purpura or ecchymosis.

Woodhead offers similar explanations of those of Adami.

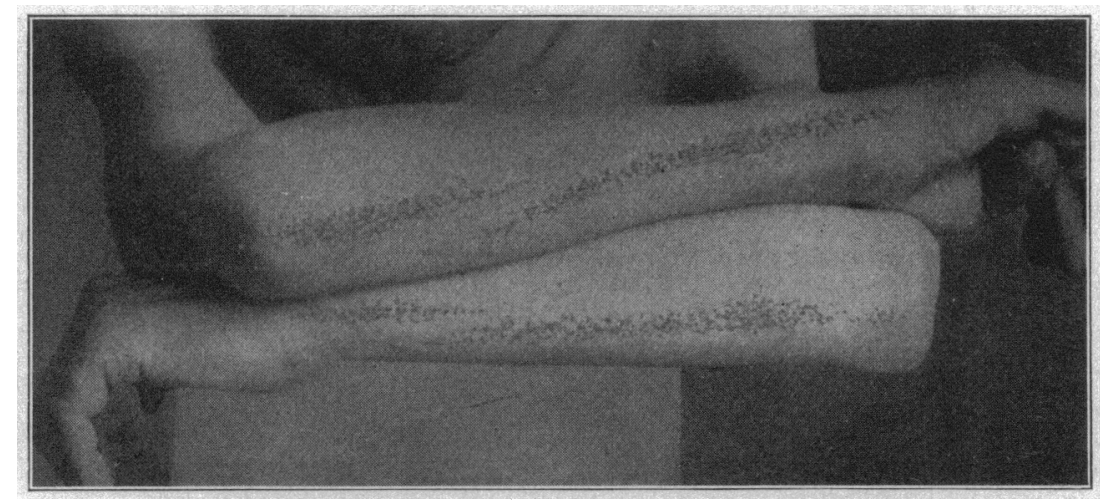

Fig. 1.--Showing purpuric areas on arms of patient G. I. (Case 3).

Muir and Ritchie ${ }^{6}$ claim that "in some cases of acute tuberculosis, when the bacilli become lodged in a capillary, the endothelial cells of its wall may proliferate, and thus a ring of nuclei may be seen round a small central thrombus."

Unna $^{\top}$ acknowledges that from the information he received from others and from the results of his own wide experience, he fails to understand how a capillary hemorrhage is brought about. He claims that it is neither by the ordinary understood rhexis or diapedisis, that capillary hemorrhages are produced. There must be, he says, a specific cause or a "blind force" which renders these small cutaneous vessels

4. Struempell's Text Book on Medicine.

5. Adami's Principles of Pathology.

6. Muir and Ritchie: Manual of Bacteriology.

7. Unna: Histopathology of the Diseases of the Skin. 
subject to hemorrhage. His arguments against the accepted theories regarding these purpurae are as follows:

1. The blood vessels of the skin are not disposed to hemorrhage.

2. The locus minoris resistantiae is in the subcutaneous vessels more than in the cutaneous.

3 . The contention that bacteria are probably the cause of hemorrhage he disproves by the fact that in phlyctenosis streptogenes, the streptococci are found in the capillaries; the same is true of the disease known as pustulosis staphylococci, but they cause necrosis of the endothelium only and not bleeding.

4. In hyaline degeneration of the endothelium he says that it produces thickened walls but no fragility; therefore there is no chance for rupture.

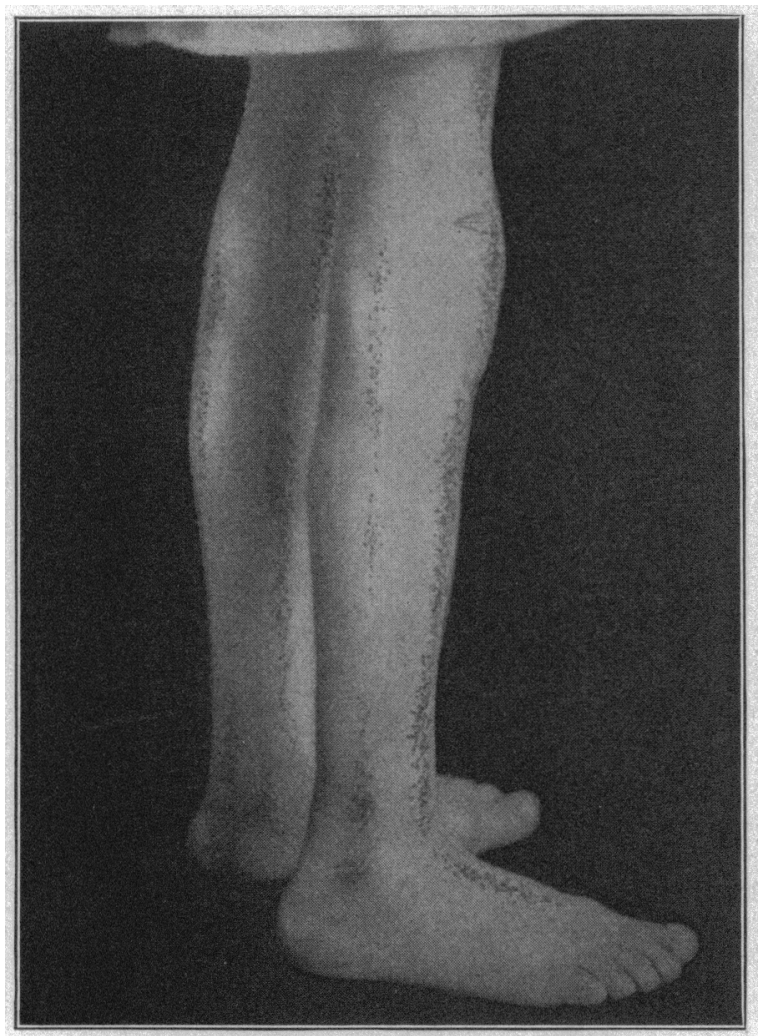

Fig. 2.-Purpuric areas on legs of patient G. I. (Case 3).

5. He also trics to disprove the theory of Rayer, who found purpura after the right iliac vein was thrombosed, with the argument that in varicose veins the tone of the vessel helps dilatation, and never rupture.

6 . The only condition of purpura in which he recognizes the complete degeneration of the vessel as being its immediate cause is in the vessel paralysis or antemortem vascular changes, where the vessel yields and ruptures.

Pratt, in Osler's Modern Medicine, among the many causes of purpura which we have already discovered, quotes a good many authorities 
who have worked on this subject from the serological point of view. This work has been done mainly at the Johns Hopkins Hospital. According to the author the probable additional causes for purpura may be a lack of fibrinogen in the blood, or a fibrinolysin, because in many cases of purpura one finds the coagulation time delayed. The authors also found that in the case of any fatty degeneration of the liver (the yellow atrophy of the liver) the cells are destroyed, lack of fibrinogen is the result of these changes, thus aiding in producing this condition. The same is true of phosphorus poisoning, which acts similarly on the liver.

They also found the blood platelets diminished in purpura, and that there is usually a leukocytosis present. Unna repudiates this very strongly.

Ewing ${ }^{8}$ claims that bacteria are found in some cases in the blood and these are isolated, as for instance: Letzeuck's Bacillus purpura, which is in turn produced in animals; Streptococcus pyogenes has been isolated in typical cases by Hanot and Luzet, Widal, Therese and Guarnieri; Staphylococcus pyogenes aureus has been isolated by Lebreton, Litton, Fischl, Adler, Lewis and Librestini; the Pneumococcus lanceolatus by Clause and Claudi.

Changes in blood were found by Carrere and Gilbert, who reported a mild case having 3,350,000 red cells, while in more severe cases the diminishing number is greater; the hemoglobin index was subnormal. They also found a leukocytosis.

Hayem $^{9}$ claims that there exists a retardation of the coagulation time, and a reduction of blood plates.

Ewing states that the clotting appeared to be abnormally rapid, as in the course of an hour the specimen in the hematocytometer, diluted 1 : 100 with 0.6 per cent. salt solution, became jelly-like. Grawitz has also found an increase in the coagulability of the blood in these cases, after repeated hemorrhages, but red cells were found rapidly diminishing, and leukocytes were normal or reduced in numbers.

Stelwagon $^{\mathbf{1 0}}$ agrees with Ewing in saying that there exists a mechanico-bacterio-toxic and chemical cause of purpura. He maintains that there must be a hyperemia and rupture or diapedesis of the vessel to produce purpura.

Hayem, who claims that the retardation of coagulability of the blood is due to the diminution of platelets, and to their destruction brought about by intestinal toxemia ; this phenomenon is also found in cachectic conditions, such as leukemia, nephritis, carcinoma and "tuber-

8. Ewing's Clinical Pathology of the Blood, 1903.

9. Hayem: Quoted in Am. Jour. Med. Sc., 1911, cxlii, by Mathew and Carpenter in Purpura Hemorrhagica.

10. Stelwagon's Diseases of the Skin, Ed. 7, 1915. 
culosis with purpura, the toxic substances of which are likely to produce profound changes in the blood, which lead to the rapid disintegration of the vessel wall, or to the slower hyaline or fatty degeneration."

John M. Cruice ${ }^{11}$ says:

Bensaude and Rivet think that purpura is not infrequently associated with tuberculosis. Of their thirty-five cases of chronic purpura hemorrhagica, seven occurred in individuals undoubtedly tuberculous, and in individuals probably tuberculous. They say that in the presence of a purpura, particularly in the recurring or chronic form, when the cause is not apparent, the clinician should by all means in his power, look for some chronic tuberculous lesion, either of the lungs, of the glands, or some other part of the body.

Brown says that purpura hemorrhagica rarely occurs in tuberculosis. In the last 1,000 cases at the Adirondack Cottage Sanitarium but three cases have occurred. This corresponds closely to my figures. Out of 1,626 ward patients at the Phipps Institute I was able to find only eight cases of purpura. Mackenzie, in 200 cases of purpura, found it associated with tuberculosis only four times. Pratt in 258 cases of purpura, both primary and secondary, found it associated with tuberculosis seven times.

Grenit attributes the cause of purpura to one of the following conditions :

1. Hepatic lesion.

2. Nervous injury.

3. Intoxication of some sort.

4. Bacteria.

5. Blood changes.

but offers no discussion or explanation thereof.

Howell ${ }^{12}$ states that he found a great diminution in the prothrombin, as in hemophilia, which retards coagulation and does not affect the antithrombin substance.

In thrombosed vessels the prothrombin is not affected but the antithrombin is diminished. In purpura hemorrhagica neither is affected.

Gaucher ${ }^{13}$ claims as follows:

1. Infective secondary purpura is met with in the course of infective diseases such as variola, scarlatina, measles, intermittent fever, pyemia, puerperal infection, ulcerative endocarditis, acute tuberculosis (of which purpura may be a premonitious phenomenon), diphtheria, enteric fever, typhus, plague, malignant jaundice, pneumonia, cerebrospinal meningitis, infective tonsillitis and gonorrhea.

2. Toxic purpura is due to the absorption of certain protein substances, but a certain predisposition is necessary for this to occur.

Herbert French" maintains that "General tuberculosis is not a common cause of purpura, and yet in a few instances extensive purpura has

11. Cruice, John M.: The Incidence of Purpura in the Course of Chronic Tuberculosis, Am. Jour. Med. Sc., 1912, cxliv, 875.

12. Howell, W. H.: Condition of Blood in Hemophilia, Thrombosis and Purpura, The Archives Int. Med., 1914, xiii, 76; absts. in Jour. Am. Med. Assn., 1914, lxii, 488.

13. Gaucher: Diseases of the Skin.

14. French, Herbert: An Index of Differential Diagnosis of Main Symptoms, William Wood \& Co., New York. 
been the first, and, for the time being, the only, symptom of an obscure illness which has ultimately turned out to be general tuberculosis. Tre patient has generally been a child, and the diagnosis has only been possible when the course of the case has been watched."

The following are the three cases which have come under observation within the last eight months. It is useless to say that they can be taken as a criterion to prove anything regarding the cause of pathogenesis of purpura, but they do show us that there is a definite interrelationship between it and tuberculosis, and possibly give a clue to its etiology.

\section{REPORT OF CASES}

Case 1.-W. S., aged 29, carpenter, Russian.

The condition of the lungs Nov. 16, 1914, was as follows:

Right lung: Dulness of both superior and middle lobes anteriorly and posteriorly, with large cavity over the same area, and moist râles all over.

Left lung: Dulness of both superior and middle lobes anteriorly and posteriorly, with amphoric breathing over the same area, and moist râles all over.

From Dec. 12 to Dec. 29, 1914, the patient showed the following additional symptoms: edema over the ankles, marked dyspnea, cyanosis, slight epistaxis, abdominal pain and nausea.

Dec. 29, 1914, patient was awake until 3 o'clock in the morning, felt nauseated and vomited coffee-colored material three times. Bowels also moved three times during the night; stools were loose and watery in consistence. Patient vomited twice in the morning, a dark greenish fluid. On examination patient was found markedly prostrated. He did not complain of anything except marked weakness. Hemorrhagic spots covered both legs, over the anterior and lateral surfaces and posteriorly. Similar hemorrhagic outlines covered the upper third of both legs and lower third of both thighs. Some spots were also seen over parts of the buttocks. They were also distributed over the inner surfaces of both forearms and upward to the midpoint of the arms. These hemorrhagic spots were confluent and covered areas 4 to 6 inches in length and about 3 inches in width. They were of a deep bluish-red hue.

The mucous membranes and the conjunctivae were negative. The chest findings were the same as on previous examinations.

From Dec. 29, 1914, to Jan. 1, 1915, the patient persistently vomited dark greenish fluid. The greater curvature of the stomach was low, reaching midway between the pubes and umbilicus, and a splashing fluid was readily made out.

From Jan. 1 to Jan. 10, 1915, the patient complained for the first time of pain in the right knee, and in both elbow joints. The purpuric rash became paler, and gradually disappeared. The face was puffed.

From Jan. 10 to Jan. 14, 1915, the puffiness grew more marked, the purpuric rash reappeared over the legs. Examination of the urine revealed an excessive amount of albumin, and numerous casts of all kinds, including a few waxy casts, and many cylindroids.

From Jan. 14 to Jan. 16, 1915, the purpuric rash gradually disappeared again. The patient became very dyspneic and cyanosed; the pulse was rapid, feeble and irregular in force. The heart beat was irregular, and a blowing 
systolic murmur was heard at the apex, which was not transmitted. The second pulmonic was accentuated.

Jan. 16, 1915, the patient's general condition was somewhat improved. $\mathrm{He}$ complained of hunger, and wished to be out of bed, but dyspnea was still marked. The pulse was small, feeble and irregular. The puffiness of face subsided. There was no edema of the extremities. At 11:15 a. $\mathrm{m}$. the patient sat up, but suddenly fell back and expired.

The blood on repeated examinations showed an average leukocyte count of 8,000 to 12,000 ; red cells of $4,500,000$ to $5,000,000$, and neutrophils 70 per cent. The hemoglobin was an average of 75 per cent. The blood culture revealed nothing.

CASE 2.-S. A., aged 28, draughtsman, American.

The condition of the lungs was as follows:

Right lung: Dulness anteriorly from apex to third interspace; posteriorly from apex to sixth spinal vertebra; also one stripe at the base. On auscultation was found bronchial breathing, with moist râles from apex to third interspace anteriorly; posteriorly bronchial breathing, with moist râles from apex to sixth vertebral spine; below that there was feeble breathing.

Left lung: There were crackles all over anteriorly; posteriorly there was dulness over the supraspinous fossa, and feeble breathing could be heard to the fifth vertebra. Below that there was hyperresonance.

An attempted artificial pneumothorax on the right side was without the desired result. May 8, 1915, the patient developed marked edema of the right lower extremity, which lasted for three days, and which was accompanied by pain in the leg. The third day his left foot became markedly edematous. There were no signs of abdominal fluid or enlargement of liver or spleen. On examination the patient showed signs of a spontaneous pneumothorax on the right side, with signs of the heart being pushed over to the left.

The roentgenogram showed that there was apparently a pneumothorax occupying the entire right thoracic cavity, pushing the collapsed right lung, as well as the mediastinum, completely to the left side.

About the left side nothing definite could be said, as it was markedly compressed and obscured by the mediastinum.

May 29, 1915, the patient was very dyspneic, showed pronounced cyanosis over the extremities, and complained of pain in swallowing. On examination evidence of fluid could be elicited at the base of the right chest, and 33 ounces of thin, turbid fluid, greenish in color, was obtained.

June 1, 1915, the general condition of the patient was very poor. There was tenderness all over the abdomen, especially over the liver, which was very large, and could be palpated as far as the umbilicus. He had also edema of the right lower extremity. His elbow and knee joints were tender. The patient showed a purpuric rash over the sacral region, over the right clavicle, anteriorly and posteriorly, over the right supraspinous fossa, and over the anterior portion of the right leg. These purpuric spots were of a deep bluish hue, which did not disappear on pressure, and which covered areas about 3 to 5 inches in length and 3 inches in width.

On five successive urinary examinations, before and after the appearance of the purpuric rash, no albumin could be found.

On two successive blood counts, before and after the appearance of the rash, the patient had an average of hemoglobin of 65 per cent.; red blood cells, $5,000,000$; white blood cells, 6,000 ; polymorphonuclear cells, 84 per cent.; small and large mononuclear cells, 16 per cent. The blood culture was found negative, and no bacilli were found in the blood. 
CASE 3.-G. I., aged 38, tailor, Russian.

The lung condition on admission was as follows:

Feb. 18, 1915, the right lung showed dulness over two upper lobes, anteriorly and posteriorly, with bronchovesicular breathing and moist râles; below that the note was hyperresonant.

The left lung showed dulness of the entire side, anteriorly and posteriorly, with bronchial breathing and moist râles all over.

April 7, 1915, the physical examination and fluoroscope showed symptoms and signs of complete right-sided pneumothorax; absent breath sound all over, except the upper third posteriorly, and orthopnea.

On inquiry the history given by the patient tells of a sudden onset at 10:30 p. m., April 5, 1915.

May 8, 1915, the patient developed a purpuric rash over the extensor and flexor surfaces of the right arm, up to the elbow, and a similar condition on the extensor surface of the left thigh. The next day the patient had a swelling of the right wrist, followed by vomiting of bile-colored mucus. This vomiting lasted for three days. The patient also had diarrhea and blood-tinged stool. He also developed edema of both feet. This condition remained unchanged until May 23, 1915, when the patient developed edema of lower extremities. The purpuric rash on the lower extremities became more confluent, and he complained of pain in the knee joint, and also dyspnea on slight exertion.

From Jan. 18 to May 5, 1915, patient's urine showed the presence of albumin, hyaline, finely granular and epithelial casts.

The blood picture before and after the onset of purpura showed that the systolic blood pressure averaged 106, and diastolic, 80 ; hemoglobin, 80 per cent.; red blood cells, 4,450,000; white blood cells, 8,000; neutrophils, 75 per cent.; small and large lymphocytes, 29 per cent. Blood culture negative. Coagulation time normal.

\section{CONCLUSION}

Clinically expressed, the purpura observed in our three cases can be grouped in the same category with the condition known as peliosis rheumatica of Schoenlein. All the patients complained of pain in the joints, which came on suddenly and simultaneously with the onset of the rash, and all the cutaneous hemorrhages were of the simple type, with the exception of a single case, in which the patient's stool showed traces of blood-evidently suggesting purpura hemorrhagica proper.

Pathologically, we cannot put our cases in one distinct type of purpura, because they show characteristics common to all the classes.

For instance, blood cultures were found negative for bacteria. The red blood cells averaged from 4,500,000 to 5,000,000; white blood cells averaged 6,000; neutrophils 80 per cent., hemoglobin averaged 65 per cent., blood platelets were normal. The time of coagulation was norcal. They all had edema of the extremities; all had diarrhea; one, in addition, showed albumin and casts in his urine; another had bloody stools; they were all prostrated; all manifested symptoms of spontaneous pneumothorax, and all showed a rash on similar locations, almost similarly distributed; all were confluent, and did not disappear on pressure. 
Therefore, the following are the facts which explain our claim:

1. According to Muir, though not found in our cases, the tubercle bacilli form colonies in the cutaneous vessels, where they act as an irritant.

2. The secreted or excreted tuberculous toxins contribute to the necrosis of the cutaneous vessels.

3. The serum extravasated into the dependent portions, either by pressure exerted on some vein or lymphatic vessel, or when this is directly due to nephritis, produces in turn a pressure on the cutaneous vessels, which may act as a mechanical factor.

4. In the later stages of pulmonary tuberculosis the viscera may undergo either hyaline, amyloid or fatty degeneration, which aids in disintegrating the endothelial cells of the blood vessels, producing necrosis of the endothelium, and rupture; the fibrinogen-forming cells in the liver are also destroyed by the morbid process, thereby producing diminution in the viscosity of the blood, and also delaying its coagulation.

To sum up: The blood picture found in our three cases does not correspond with that found by the various authors quoted in our review of the subject.

But we did find, in these three cases, sufficient factors to warrant us in claiming that there is a relationship between purpura and tuberculosis.

They all showed the symptoms of intestinal toxemia, of amyloid degeneration, of the mechanical factors, and of general toxemia.

As we have seen in our review, one writer would lay stress on one factor as being the cause of purpura, another would find a different cause. In our cases we found a few causes which strengthen our belief that purpura can be found more often in tuberculosis than has been reported.

I am indebted to Dr. M. Fishberg and Dr. S. Wachsmann for their aid, encouragement and courtesy in offering their material. 